\title{
Comparison of induction therapy with anti- thymocyte/antilymphocyte globulin or anti-IL-2 receptor antibody in pediatric kidney transplantation: a systematic review and meta- analysis
}

\section{Xiaohong Lin}

Sun Yat-sen University First Affiliated Hospital

Miaohan Deng ( $\nabla$ dengmh6@mail3.sysu.edu.cn )

Sun Yat-sen University First Affiliated Hospital https://orcid.org/0000-0003-2058-8764

\section{Xitao Hong}

Sun Yat-sen University First Affiliated Hospital

\section{Weiqiang Ju}

Sun Yat-sen University First Affiliated Hospital

\section{Maogen Chen}

Sun Yat-sen University First Affiliated Hospital

\section{Research article}

Keywords: anti-thymocyte globulin, antilymphocyte globulin, anti-IL-2, pediatric kidney transplantation, meta-analysis

Posted Date: June 18th, 2021

DOl: https://doi.org/10.21203/rs.3.rs-629420/v1

License: (c) (i) This work is licensed under a Creative Commons Attribution 4.0 International License. Read Full License 


\section{Abstract}

\section{Background}

Anti-IL-2 antibody (basiliximab or daclizumab) and anti-thymocyte globulin (ATG)/antilymphocyte globulin (ALG) are widely used as induction agents in pediatric kidney transplantation. However, which of them benefits patients more remains unknown.

\section{Methods}

Online databases were searched to identify controlled clinical studies that compared anti-IL-2 with ATG/ALG for induction therapy in pediatric kidney transplantation. Odds ratios (OR) and $95 \%$ confidence interval $(\mathrm{Cl})$ were chosen to compare the gathered data. Review Manager 5.4 was applied to identify differences in outcomes between the two agents.

Results

Five retrospective cohort studies were included, enrolling a total of 2510 pediatric patients, 1152 (45.7\%) of whom had received ATG/ALG therapy and 1370 (54.3\%) of whom received anti-IL-2. According to the pooled results, no differences were seen between anti-IL-2 and ATG/ALG regarding the delayed graft function (DGF) rate (odds ratio (OR) 1.1; $95 \%$ confidence interval $(\mathrm{Cl}) 0.36-3.39 ; \mathrm{P}=0.85$ ), 6-month acute rejection rate (OR $0.80 ; 95 \% \mathrm{Cl} 0.62-1.03 ; \mathrm{P}=0.09)$, 1 -year acute rejection rate (OR $0.98 ; 95 \% \mathrm{Cl} 0.78-$ $1.24 ; \mathrm{P}=0.88)$, 1-year graft survival rate $(\mathrm{OR} 1.37 ; 95 \% \mathrm{Cl} 0.91-2.06 ; \mathrm{P}=0.13), 1$-year patient survival rate (OR 0.86; $95 \% \mathrm{Cl} 0.40-1.86 ; \mathrm{P}=0.70$ ) and 1-year post-transplantation lymphoproliferative disorder (PTLD) rate (OR 0.30; $95 \% \mathrm{Cl} 0.03-3.16 ; \mathrm{P}=0.32)$.

\section{Conclusions}

Anti-IL-2 have the same efficacy and safety as ATG/ALG in transplant induction therapy. However, as most of included studies were small-scale retrospective studies, further studies are needed to identify an optimal choice with certain.

\section{The analysis had been registered in PROSPERO and the registration ID is CRD42021237561.}

Comparison of induction therapy with anti-thymocyte/antilymphocyte globulin or anti-IL-2 receptor antibody in pediatric kidney transplantation: a systematic review and meta-analysis

\section{Introduction:}

Kidney allograft transplantation is currently the optimal option for children with end-stage renal disease to preserve their growing potential[1, 2]. However, several complications may follow, including rejection, infection and neoplasm, which can contribute to graft loss. In addition to the transplantation operation itself, perioperative immunosuppressive therapy also plays an important role in impacting prognosis. It 
usually starts at the moment of transplantation, with induction regimens that aim at preventing acute rejection[3]. There are several inductive agents, either T cell nondepleting (anti-IL-2R antibody, namely, daclizumab and basiliximab) or depleting (thymoglobulin and alemtuzumab) agents. Thymoglobulin, listed in the earliest immunosuppressive protocols, was clinically effective but lacked immunosuppressive specificity[4]. By contrast, basiliximab and daclizumab are chimeric and humanized IL-2Ra inhibitors, respectively[5]. Though the North American Pediatric Renal Transplant Cooperative Study suggests that the induction of ATG leads to less acute rejection and more graft survival than does no induction[6], it is considered to increase the risk of infection and PTLD in pediatric kidney allografts [1, 7]. As a result, trends in induction therapy have shifted away to anti-IL-2R antibodies, which are thought to have lower immunosuppressive burden and better tolerability in clinical practice[1, 8, 9]. However, consensus regarding which agent benefits pediatric patients has not yet been reached. Although a few clinical studies have compared them in pediatric kidney transplantation, their results are varied. Therefore, we conducted a meta-analysis, gathered the relevant results and hoped to reach a comprehensive answer. The analysis had been registered in PROSPERO and the registration ID is CRD42021237561.

\section{Methods:}

\section{Search Strategy}

In July 2020, Medline (PubMed), Embase, Web of Science, clinicaltrials.gov, and the Cochrane Central Register of Controlled Trials were searched for studies between 2000 and 2020 comparing anti-IL-2R antibodies (basiliximab or daclizumab) with ATG/ALG in pediatric kidney transplantation. The search terms of Medline were set as follows: "((interleukin 2 receptor antibody[All Fields]) OR (anti-CD25[All Fields]) OR (basiliximab[MeSH Terms]) OR (daclizumab[MeSH Terms])) AND ((Antilymphocyte Serum[MeSH Terms]) OR rATG[All Fields]) AND (kidney transplantation[MeSH Terms]) AND (child[Mesh Terms] OR pediatric[All Fields]))"; those of Embase were as follows: " ('basiliximab'/exp OR 'daclizumab'/exp OR 'interleukin 2 receptor alpha'/exp) AND ('thymocyte antibody'/exp) AND ('kidney transplantation'/exp) AND ('child'/exp OR 'adolescent'/exp OR 'pediatric surgery'/exp)". In other databases, the search terms were as follows: 'Kidney Transplantation' AND 'Antilymphocyte Serum', 'Daclizumab', 'Basiliximab', 'Adolescent', 'child'. The searched was not limited to English, though none of the studies in other languages was included. Reference lists from all available reviews were searched manually.

\section{Inclusion and exclusion criteria}

The included studies needed to meet the following criteria: (1) enrollment of patients younger than 21 years old (children and adolescents); (2) controlled studies comparing either basiliximab or daclizumab versus ATG for induction therapy in kidney transplantation; (3) follow-up time $\geq 12$ months; and (4) assessment of at least 1 of the following outcomes: delayed graft function rate, 1-year acute rejection rate, 1-year graft survival rate, 1-year patient survival rate, and the rate of PTLD. The exclusion criteria 
were as follows: (1) multiorgan, combined organ transplantation; and (2) mixed-aged studies without specific groups for those younger than 21 years old.

\section{Data Extraction}

One investigator reviewed the titles and abstracts of available articles. The articles reaching the inclusion criteria were retrieved for full-text assessment. The following data were extracted: first author, country of author, year of publication, interventions, dosage of drugs, number of participants and outcome measures. In the studies that included a third group other than anti-IL-2R antibodies or ATG/ALG, only data of the 2 groups were extracted[9-11].

\section{Statistical Analysis}

The data analysis was performed using the statistical software Review Manager Version 5.4 (The Cochrane Collaboration, Oxford, United Kingdom)(RRID:SCR_003581).

All of the included data were dichotomous variables. The compared outcomes are presented using odds ratios (ORs). The OR value was considered statistically significant if $\mathrm{P}<0.05$ and the $95 \%$ confidence interval $(\mathrm{Cl})$ did not include the value one.

The quality of the studies was evaluated using the Newcastle-Ottawa Scale[12]. A study can be awarded a maximum of one star for each numbered item within the selection and outcome categories. $\mathrm{A}$ maximum of two stars can be given for comparability. Studies scoring more than five stars were considered to be of high quality.

Heterogeneity was evaluated using the chi-square test. The $\mathrm{P}$ value and value was used to evaluate statistical heterogeneity. If the value is $<50 \%$ and the $P$ value is $\geq 0.1$, it is considered to have nonsignificant heterogeneity. If the value is $\geq 50 \%$ and the $P$ value is $<0.1$, the data would be thought to have significant heterogeneity. As the studies varies in investigators, time and locations, a random-effects model was used for all the studies. As only 6 studies were included, publication bias was not assessed.

\section{Results:}

\section{Description of the studies}

In total, 462 articles were generated by using the search strategy above. After the titles and abstracts were scanned, 9 articles were obtained for full-text assessment. Three of them were excluded due to the absence of grouped comparisons. Ultimately, 6 articles enrolling 2522 patients were included[9-11, 13-15]. Among the patients, 1152 (45.7\%) had received ATG/ALG therapy, and 1370 (54.3\%) had received anti-IL$2 \mathrm{R}$ antibodies. The process of study selection is presented in Figure 1. The main features of included study are shown in Table 1. The summary of the outcomes is shown in Table 2.

\section{Meta-analysis of anti-IL-2R antibody versus ATG/ALG group}




\section{Delayed graft function rate}

Five studies reported the rate of DGF. The pooled results revealed no significant differences between the two groups, with a pooled OR value of $1.1(95 \% \mathrm{Cl} 0.36-3.39 ; \mathrm{P}=0.07)$. As the chi-square test suggested the existence of heterogeneity $(=8.76, p=0.07,=54 \%)$, following sensitivity analysis was conducted. We found that the only prospective study in our analysis was the source of heterogeneity, which showed a reduction in the DGF rate in the ATG group[15]. This outcome is shown in Figure 2(a).

\section{6-month acute rejection rate}

Five studies reported the rate of 6-month acute rejection, involving 1365 patients inducted with anti-IL-2R antibodies and 1147 with ALG/ATG. No significant heterogeneity was identified among them $(=4.61, p=$ $0.20,=35 \%)$. Three of them found a superior result in the anti-IL-2R group, but none reported any statistical significance. The combined 6-month acute rejection rate was $10.3 \%$ in the anti-IL-2R antibody group and $12.7 \%$ in the ATG/ALG group (none of significance), with a pooled OR value of $0.80(95 \% \mathrm{Cl}$ 0.62 -1.03; $P=0.09)$. This outcome is presented in Figure 2(b).

\section{1-year acute rejection rate}

Four studies reported the rate for the 1-year acute rejection rate, with only 1 study reporting better outcomes for anti-IL-2R than for ATG/ALG. No significant heterogeneity was found $(=2.22, p=$ $0.33,=10 \%)$. None of the single studies showed a significant difference, and the combined incidence was $13.5 \%$ using anti-IL-2R and $13.7 \%$ using ATG/ALG, indicating no significance. The pooled OR value was $0.98(95 \% \mathrm{Cl} 0.78-1.24 ; \mathrm{P}=0.88)$. This outcome is shown in Figure 2(c).

\section{1-year graft survival rate}

Six studies reported the rate of 1-year graft survival, enrolling 1370 recipients using anti-IL-2R and 1152 using ATG/ALG. None of the studies reported significant differences between the two groups. None of the significant heterogeneity was identified $(=2.45, p=0.78,=0 \%)$. The pooled 1 -year graft survival rate was $96.8 \%$ in the anti-IL-2R group and $95.6 \%$ in the ATG/ALG group (none of significance), with a pooled OR value of $1.37(95 \% \mathrm{Cl} 0.91-2.06 ; \mathrm{P}=0.78)$. This outcome is shown in Figure $2(\mathrm{~d})$.

\section{1-year patient survival rate}

Six studies, with 1370 patients in the anti-IL-2R antibody group and 1152 in the ATG/ALG group, reported a 1-year patient survival rate. None of the significant heterogeneity was identified $(=0.46, p=0.79,=0 \%)$. None of the studies reported significant differences between the two groups. The combined 1-year patient survival rate of both groups was $99 \%$. The pooled $\mathrm{OR}$ value was $0.86(95 \% \mathrm{Cl} 0.40-1.86 ; \mathrm{P}=0.70)$. This outcome is shown in Figure 2(e).

1-year PTLD rate 
Four studies reported the rate of PTLD, including 99 patients in the anti-IL-2R antibody group and 86 in the ATG/ALG group. There was no significant heterogeneity identified among the 4 studies $(=0.01, p=$ $0.94,=0 \%)$. Only 2 studies reported cases of PTLD, both in the ATG/ALG group. However, no significant difference was identified. The pooled OR value was $0.30(95 \% \mathrm{Cl} 0.03-3.16 ; \mathrm{P}=0.32)$. This outcome is shown in Figure 2(f).

\section{Discussion:}

According to The Scientific Registry of Transplant Recipients (SRTR), the overall 5-year patient survival of pediatric kidney transplantation was promising $98.4 \%$ in USA[16]. The use of inductive therapy has significantly contributed to this outcome. In 2018 , it had been applied in approximately $98.7 \%$ of the recipients [16]. Though traditionally favored, ATG as induction is capable of interfering with multiple immune effector cells and inducing various biological effects[17]. It is associated with cytokine release syndrome, thrombocytopenia, lymphopenia and other conditions [17]. Compared with ATG, IL-2R antibodies, with a low prevalence of side effects, tend to be increasingly recommended for low or average immunologic risk patients[17-19]. However, in the USA transplant centers, T-cell depleting agents (including ATG/ALG and alemtuzumab) were preferred regardless of immunologic risk judged by calculated penal reactive antibody $[16,20]$. According to SRTR, use of T-cell depleting agents had reached $63.6 \%$, and IL-2R antibodies remained at $35.1 \%$ by $2018[16]$. ATG was the most widely used of T-cell depleting agents $[21,22]$. However it was commonly used, the consensus on which agent is more beneficial has not yet been reached[ $[8,22]$. Therefore, we performed this meta-analysis to explore whether anti-IL-2R antibodies or ATG/ALG can lead to better clinical outcomes.

Acute rejection is a strong risk factor for graft loss and is considered one of the major indicators in the relevant literature $[1,8]$. ATG and anti-IL-2R antibodies were both proven to be protective from it, but which is optimal is uncertain[23-26]. In our study, we did not find a significant difference between them in preventing 6-month or 1-year acute rejection. This finding was also in line with most studies in adults [27-29]. DGF is another problem defined as the requirement for dialysis within 1 week after allograft[30]. Although many patients can recover within a few days, it may increase the complexity of care and make the diagnosis of acute rejection difficult [31]. In our study, pooled outcomes, including 4 retrospective studies and 1 small-sample-size prospective study, suggested that ATG/ALG and anti-IL-2R antibodies share the same efficacy. However, with significant heterogeneity, this outcome is not robust enough. If we exclude the single prospective study, the heterogeneity would disappear, and anti-IL-2R antibodies would manifest significantly better outcomes than the ATG/ALG group. In fact, DGF has complex causes, including ischemic reperfusion injury and systemic inflammatory response syndrome[15]. Its strong risk factors, including donor qualities and donor cause of death, were not addressed in the existing retrospective studies[31]. In contrast, the single prospective study kept the donor type, donor age, cold ischemic time and other suspected risk factors strictly controlled. Although this study, suggesting a lower ratio of DGF in the ATG/ALG group, may offer some references, its extremely small sample size and nonrandomized design poses a question regarding its validity. In fact, large-scale randomized controlled trials (RCTs) comparing ATG and basiliximab have already been conducted in adults and tended to deny the 
significant difference in the DGF rate between them[27]. Large-scale RCTs in children are desired. Although many trials have been conducted in adults, pediatric populations specifically are rarely examined, making it difficult to explore specific complications. For example, recipients aged under 10 are more likely to suffer from PTLD, a more serious and life-threatening complication than others[32]. Although ATG/ALG was widely believed to be associated with an increased incidence of PTLD and all cases in our study occurred in the ATG/ALG group, a significant difference was not observed[7]. However, due to the small sample size of the included studies, more supportive evidence is needed. The insufficiency of follow-up time also made our conclusion suspicious, as PTLD may occur within a long period after operation.

Our analysis has some limitations. First, the infection rate is an important indicator and a strong risk factor for graft loss but was excluded from our analysis due to unavailable data. Second, data on longterm survival are lacking, although it may be one of the key concerns of clinical doctors. We hope that studies with longer follow-up time will be conducted on children. Third, our analysis was seriously limited by the small sample size or the retrospective study design of the enrolled studies. Collectively, most studies focusing on pediatric renal transplantation share the same weakness, that is, retrospective singlecenter studies with small sample sizes [33]. 5 out of 6 studies included were carried out in North American, which limited the reference value of our studies[9-11, 13, 15]. In retrospective studies, it is difficult to keep some suspected factors, such as race, dosage and maintenance therapy in control, which may exert an important influence on the outcome[9, 18,34]. Some of the retrospective studies used multiple regression model [10] or kept most of the baseline characteristics matched at the beginning[9, $14]$ for compensation, but others had not $[11,13]$. The only non-RCT in our study was also limited by its small sample size of 12 patients[11]. Last but not the least, there exists a significant bias in our study that ATG was typically reserved for immunologically higher-risk patients in practice[16, 33, 34]. Therefore, there may be higher-risk recipients benefiting from a depleting antibody and lower-risk recipients that do not need a depleting antibody. With the lack essential immunological risk data of each study, the result needs to be treated with caution.

In conclusion, anti-IL-2R shares equivalent short-term efficacy and safety with ATG/ALG according to available data. Though difficult to start, prospective, large-scale clinical controlled trials are needed to persuasively elucidate which is the optimal choice for pediatric recipients.

\section{Declarations}

Funding: This work was supported by the National Natural Science Foundation of China (81401324 and 81770410), Guangdong Basic and Applied Basic Research Foundation (2020A1515011557, 2020A1515010903), Science and Technology Planning Project of Guangdong Province (2016A020215048), Guangdong Provincial Key Laboratory of Organ Donation and Transplant Immunology (2020B1212060026), Guangdong Provincial International Cooperation Base of Science and Technology (Organ Transplantation) (2015B050501002), China. 
Conflicts of interest/Competing interests: The authors have no relevant financial or non-financial interests to disclose.

Availability of data and material: The data underlying this article are available in reference No.9 at http://doi.org/10.1111/j.1399-3046.2007.00764.x; reference No. 10 at http://doi.org/10.1111/j.13993046.2010.01335.x; reference No. 11 at http://doi.org/10.1016/j.transproceed.2011.09.101; reference No. 13 at http://doi.org/10.1016/s0041-1345(01)02354-5; reference No. 14 at http://doi.org/10.1093/ndt/17.7.1304; reference No.15 at http://doi.org/ 10.1016/j.transproceed.2009.06.037

Code availability: The data analysis was performed using the statistical software Review Manager Version 5.4 (The Cochrane Collaboration, Oxford, United Kingdom) (RRID:SCR_003581).

Authors' contributions XHL wrote the article and takes responsibility for the integrity of the work as a whole. CMG conceived the study and participated in its design. DMH participated in the data collection. XTH and WQJ performed critical revision of the article.

Ethics approval: This is an meta-analysis based on published data. No ethical approval is required.

Consent to participate: This is an meta-analysis based on published data. No participate consent is required.

Consent for publication: This is an meta-analysis based on published data. No publication consent is required.

\section{References}

1. Swiatecka-Urban A. Anti-Interleukin-2 Receptor Antibodies for the Prevention of Rejection in Pediatric Renal Transplant Patients. Pediatric Drugs, 2003. 5(10): 699-716. https://doi.org/ 10.2165/00148581-200305100-00005.

2. Sudan D, Bacha EA, John E, Bartholomew A. What's New in Childhood Organ Transplantation 2007. 28(12): 439-53. https://doi.org/ 10.1542/pir.28-12-439 \%J Pediatrics in Review.

3. Agarwal A, Shen LY, Kirk AD. The role of alemtuzumab in facilitating maintenance immunosuppression minimization following solid organ transplantation. Transpl Immunol, 2008. 20(1-2): 6-11. https://doi.org/ 10.1016/j.trim.2008.09.003.

4. Swiatecka-Urban A. Anti-interleukin-2 receptor antibodies for the prevention of rejection in pediatric renal transplant patients: current status. Paediatr Drugs, 2003. 5(10): 699-716. https://doi.org/ 10.2165/00148581-200305100-00005.

5. Garcia CD, Barros VR, Schneider L, Alves MD, Silveira CG, Garcia VD. IL-2 antibody induction and the outcome of pediatric renal transplants. Transplant Proc, 2002. 34(7): 2914-5. https://doi.org/ 10.1016/s0041-1345(02)03486-3. 
6. Benfield MR, McDonald R, Sullivan EK, Stablein DM, Tejani A. The 1997 Annual Renal Transplantation in Children Report of the North American Pediatric Renal Transplant Cooperative Study (NAPRTCS). Pediatr Transplant, 1999. 3(2): 152-67. https://doi.org/ 10.1034/j.13993046.1999.00011.x.

7. Kim S, Webster AC, Craig JC. Current trends in immunosuppression following organ transplantation in children. Curr Opin Organ Transplant, 2013. 18(5): 537-42. https://doi.org/ 10.1097/MOT.0b013e3283651b35.

8. Di Filippo S. Anti-IL-2 receptor antibody vs. polyclonal anti-lymphocyte antibody as induction therapy in pediatric transplantation. Pediatr Transplant, 2005. 9(3): 373-80. https://doi.org/ 10.1111/j.13993046.2005.00303.x.

9. Baron PW, Ojogho ON, Yorgin P, Sahney S, Cutler D, Ben-Youssef R, Baqai W, Weissman J, Franco E, Zuppan C, Concepcion W. Comparison of outcomes with low-dose anti-thymocyte globulin, basiliximab or no induction therapy in pediatric kidney transplant recipients: a retrospective study. Pediatr Transplant, 2008. 12(1): 32-9. https://doi.org/ 10.1111/j.1399-3046.2007.00764.x.

10. Sampaio MS, Poommipanit N, Kuo HT, Reddy PN, Cho YW, Shah T, Bunnapradist S. Induction therapy in pediatric kidney transplant recipients discharged with a triple drug immunosuppressive regimen. Pediatr Transplant, 2010. 14(6): 770-8. https://doi.org/ 10.1111/j.1399-3046.2010.01335.x.

11. Velez C, Zuluaga G, Ocampo C, Aristizabal A, Serna LM, Serrano Gayubo AK, Florez JA, Zuleta JJ. and J.J. Vanegas Ruiz, Clinical description and evolution of renal transplant pediatric patients treated with alemtuzumab. Transplant Proc, 2011. 43(9): 3350-4. https://doi.org/ 10.1016/j.transproceed.2011.09.101.

12. Wells GA, O'Connell BS,D, Peterson J, Welch V, Losos M. P Tugwell,. The Newcastle-Ottawa Scale (NOS) for assessing the quality of nonrandomised studies in meta-analyses. Available from: http://www.ohri.ca/programs/clinical_epidemiology/oxford.asp.

13. Acott PD, Lawen J, Lee S, Crocker JF. Basiliximab versus ATG/ALG induction in pediatric renal transplants: comparison of herpes virus profile and rejection rates. Transplant Proc. 2001;33:7-8. p. 3180-3. https://doi.org/ 10.1016/s0041-1345(01)02354-5.

14. Clark G, Walsh G, Deshpande P, Koffman G. Improved efficacy of basiliximab over antilymphocyte globulin induction therapy in paediatric renal transplantation. Nephrology Dialysis Transplantation. 2002;17(7):1304-9. DOI 10.1093/ndt/17.7.1304.

15. Vilalta R, Lara E, Madrid A, Chocron S, Vazquez A, Casquero A, Nieto J. Delayed graft function is reduced with antithymocyte globulin induction in pediatric kidney transplantation. Transplant Proc, 2009. 41(6): 2373-5. https://doi.org/ 10.1016/j.transproceed.2009.06.037.

16. Hart A, Smith JM, Skeans MA, Gustafson SK, Wilk AR, Castro S, Foutz J, Wainright JL, Snyder JJ, Kasiske BL, Israni AK, OPTN/SRTR 2018 Annual Data Report: Kidney. 2020. 20(s1): p. 20-130. https://doi.org/ 10.1111/ajt.15672.

17. Coelho T, Tredger M, Dhawan A. Current status of immunosuppressive agents for solid organ transplantation in children. Pediatr Transplant, 2012. 16(2): 106-22. https://doi.org/ 10.1111/j.1399- 
3046.2012.01644.x.

18. Sharma A, Ramanathan P, Fisher. Pediatric kidney transplantation: a review. Transplant Research Risk Management, 2013. https://doi.org/ 10.2147/trrm.S34043.

19. Haasova M, Snowsill T, Jones-Hughes T, Crathorne L, Cooper C, Varley-Campbell J, Mujica-Mota R, Coelho H, Huxley N, Lowe J, Dudley J, Marks S, Hyde C, Bond M, Anderson R. Immunosuppressive therapy for kidney transplantation in children and adolescents: systematic review and economic evaluation. Health Technol Assess, 2016. 20(61): 1-324. https://doi.org/ 10.3310/hta20610.

20. Santos AH Jr, Li Y, Alquadan K, Ibrahim H, Leghrouz MA, Akanit U, Womer KL, Wen X. Outcomes of induction antibody therapies in the nonbroadly sensitized adult deceased donor kidney transplant recipients: a retrospective cohort registry analysis. Transpl Int, 2020. 33(8): 865-77. https://doi.org/ 10.1111/tri.13583.

21. Cai J, Terasaki PI. Induction immunosuppression improves long-term graft and patient outcome in organ transplantation: an analysis of United Network for Organ Sharing registry data.

Transplantation, 2010. 90(12): 1511-5. https://doi.org/ 10.1097/TP.0b013e3181fecfcb.

22. Laftavi MR, Sharma R, Feng L, Said M, Pankewycz O. Induction therapy in renal transplant recipients: a review. Immunol Invest, 2014. 43(8): 790-806. https://doi.org/ 10.3109/08820139.2014.914326.

23. Webster AC, Ruster LP, McGee R, Matheson SL, Higgins GY, Willis NS, Chapman JR, Craig JC. Interleukin 2 receptor antagonists for kidney transplant recipients. Cochrane Database Syst Rev, 2010. 2010(1): Cd003897. https://doi.org/ 10.1002/14651858.CD003897.pub3.

24. Woodroffe R, Yao GL, Meads C, Bayliss S, Ready A, Raftery J, Taylor RS, Clinical and costeffectiveness of newer immunosuppressive regimens in renal transplantation: a systematic review and modelling study. Health Technol Assess, 2005. 9(21): p. 1-179, iii-iv. https://doi.org/ 10.3310/hta9210.

25. Webster A, Pankhurst T, Rinaldi F, Chapman JR, Craig JC. Polyclonal and monoclonal antibodies for treating acute rejection episodes in kidney transplant recipients. Cochrane Database Syst Rev, 2006(2): p. Cd004756. https://doi.org/ 10.1002/14651858.CD004756.pub3.

26. Hill P, Cross NB, Barnett AN, Palmer SC, Webster AC. Polyclonal and monoclonal antibodies for induction therapy in kidney transplant recipients. Cochrane Database Syst Rev, 2017. 1(1): p. Cd004759. https://doi.org/ 10.1002/14651858.CD004759.pub2.

27. Liu Y, Zhou P, Han M, Xue CB, Hu XP, Li C. Basiliximab or antithymocyte globulin for induction therapy in kidney transplantation: a meta-analysis. Transplant Proc, 2010. 42(5): 1667-70. https://doi.org/ 10.1016/j.transproceed.2010.02.088.

28. Wang K, Xu X, Fan M. Induction therapy of basiliximab versus antithymocyte globulin in renal allograft: a systematic review and meta-analysis. Clin Exp Nephrol, 2018. 22(3): 684-93. https://doi.org/ 10.1007/s10157-017-1480-z.

29. Hwang SD, Lee JH, Lee SW, Park KM, Kim JK, Kim MJ, Song JH. Efficacy and Safety of Induction Therapy in Kidney Transplantation: A Network Meta-Analysis. Transplant Proc, 2018. 50(4): 987-92. https://doi.org/ 10.1016/j.transproceed.2018.01.022. 
30. Troppmann C, Gillingham KJ, Benedetti E, Almond PS, Gruessner RW, Najarian JS, Matas AJ. Delayed graft function, acute rejection, and outcome after cadaver renal transplantation. The multivariate analysis. Transplantation, 1995. 59(7): 962-8. https://doi.org/ 10.1097/00007890-19950415000007.

31. Butler T, Hayde N. Impact of Induction Therapy on Delayed Graft Function Following Kidney Transplantation in Mated Kidneys. Transplant Proc, 2017. 49(8): 1739-42. https://doi.org/ 10.1016/j.transproceed.2017.06.032.

32. Rausch L, Koenecke C, Koch HF, Kaltenborn A, Emmanouilidis N, Pape L, Lehner F, Arelin V, Baumann $\mathrm{U}, \mathrm{Schrem} \mathrm{H}$. Matched-pair analysis: identification of factors with independent influence on the development of PTLD after kidney or liver transplantation. Transplant Res, 2016. 5: 6. https://doi.org/ 10.1186/s13737-016-0036-1.

33. Moudgil A, Puliyanda D. Induction Therapy in Pediatric Renal Transplant Recipients. Pediatric Drugs, 2007. 9(5): 323-41. https://doi.org/ 10.2165/00148581-200709050-00005.

34. Crowson CN, Reed RD, Shelton BA, MacLennan PA, Locke JE. Lymphocyte-depleting induction therapy lowers the risk of acute rejection in African American pediatric kidney transplant recipients. Pediatr Transplant, 2017. 21(1). https://doi.org/ 10.1111/petr.12823.

\section{Tables}

Due to technical limitations, table 1,2 is only available as a download in the Supplemental Files section.

\section{Figures}




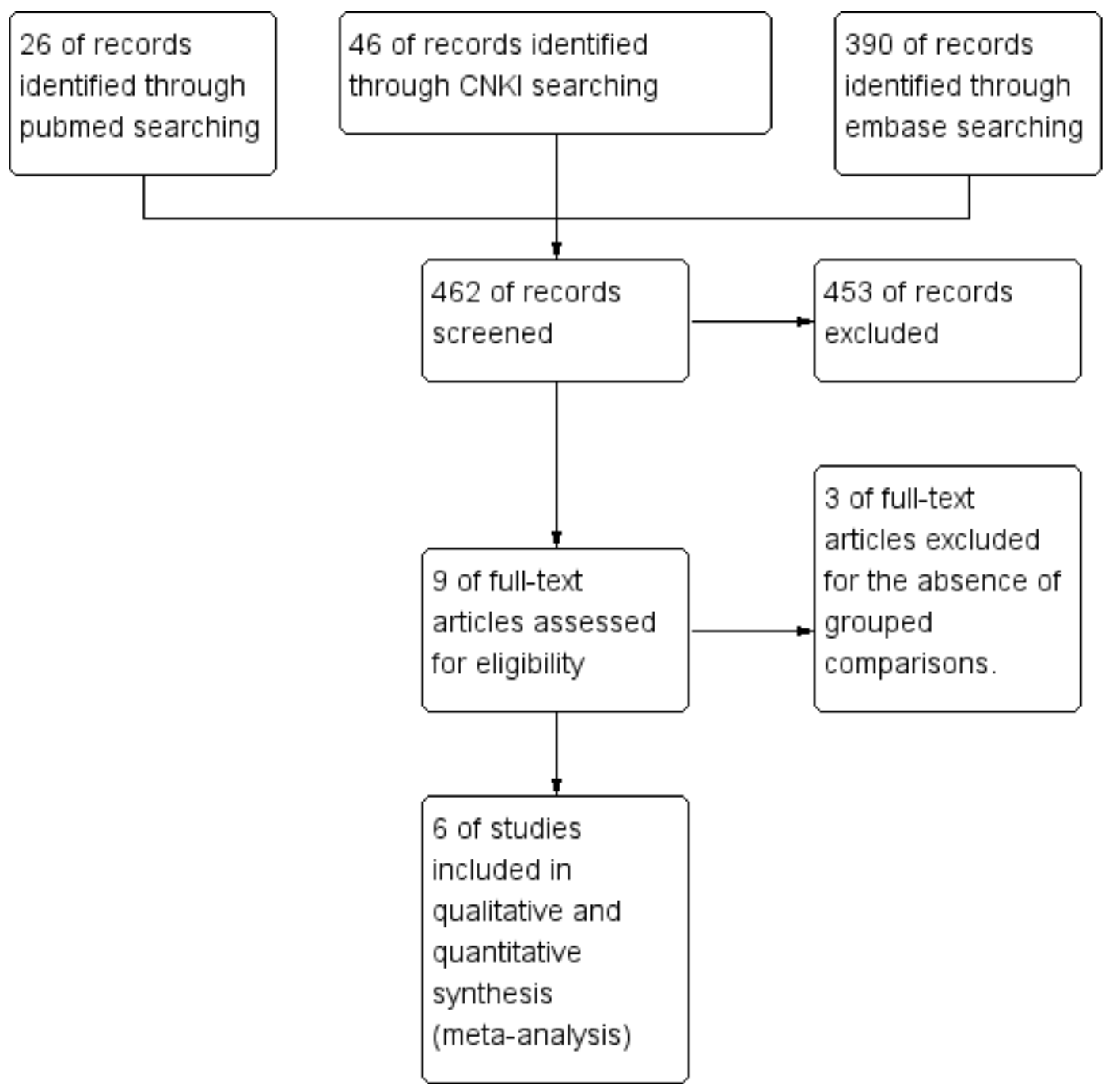

\section{Figure 1}

The flow diagram of the search process 


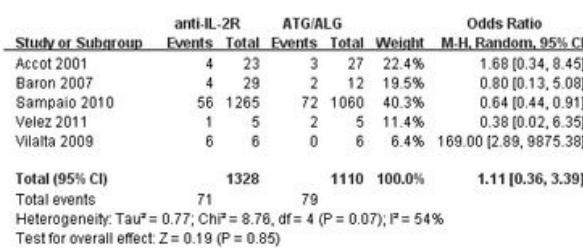

Figure 2(a)

anti-ll-2R ATG/ALG $\begin{array}{lllllll}\text { Accot } 2001 & 3 & 23 & 9 & 27 & 12.3 \% & 0.30[0.07 .1 .2\end{array}$ $\begin{array}{llllllll}\text { Baron } 2007 & 5 & 29 & 0 & 12 & 3.4 \% & 5.6110 .29,109.85\end{array}$ $\begin{array}{lllllll}\text { Clark } 2002 & 19 & 42 & 26 & 42 & 25.9 \% & 0.51[0.21,1.21\end{array}$ \begin{tabular}{lllllll}
\hline 113 & 1265 & 110 & 1060 & $58.4 \%$ & $0.85[0.64,1.12$
\end{tabular} vilalta 2009 Total $(95 \%$ CI)

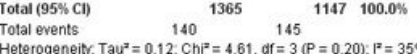
Heterogeneity $\mathrm{Tau}^{z}=0.12 ; \mathrm{Ch}^{z}=4.61$, df $=3(P=0.20) ; \mathrm{I}^{z}=35 \%$
Test for overall effect $Z=1.26(P=0.21)$
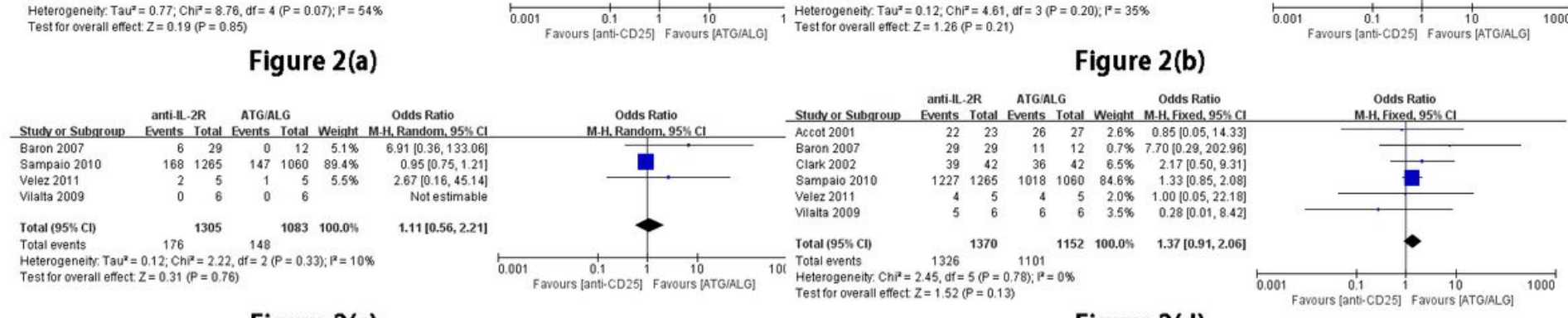

Figure 2(c)

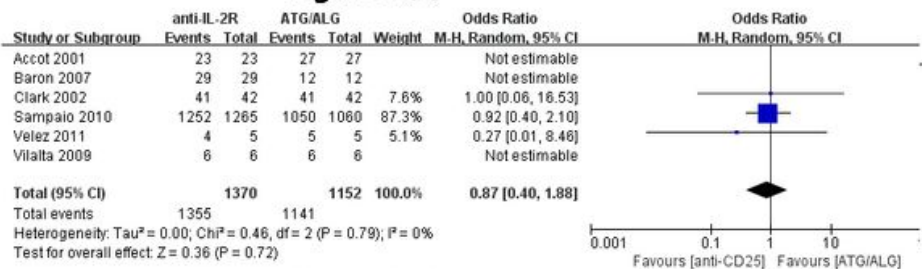

Figure 2(e)
Figure 2(b)

Figure 2(d)
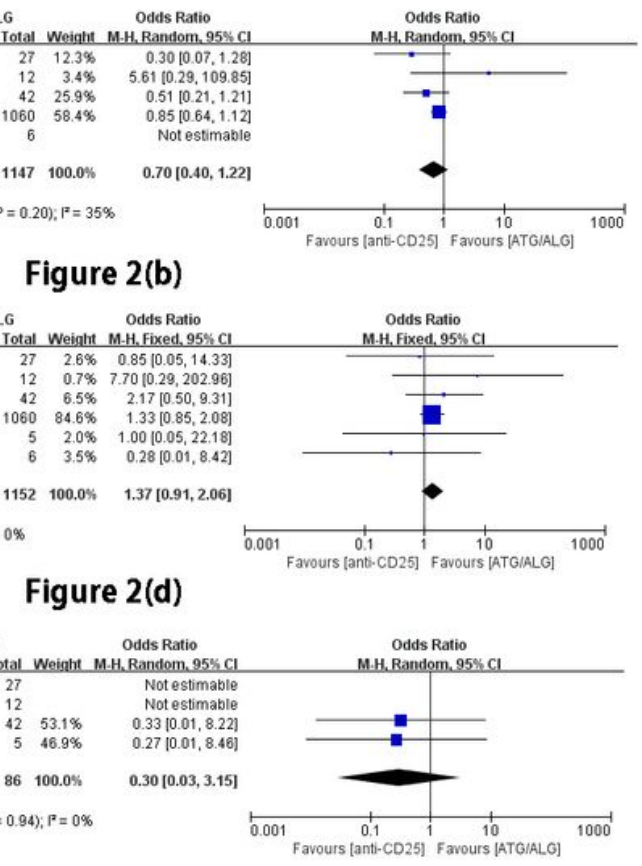

Figure 2(f)

\section{Figure 2}

The outcomes were shown as forest plots. (a)1-year DGF comparison. (b) 6-month acute rejection comparison. (c)1-year acute rejection comparison. (d) 1-year graft survival comparison. (e) 1-year patient survival comparison. (f) 1-year PTLD comparison.

\section{Supplementary Files}

This is a list of supplementary files associated with this preprint. Click to download.

- Table1.doc

- Table2.doc 